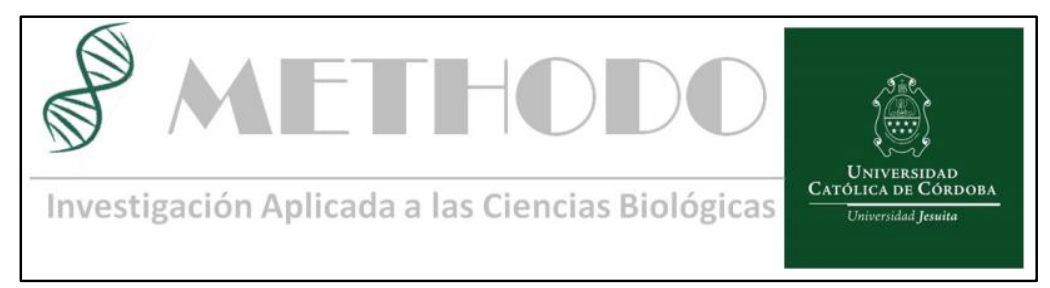

CASO CLINICO Methodo 2018;3(1): 23-26

DOI: $10.22529 / \mathrm{me} .2018 .3(1) 06$

\title{
Una presentación atípica de disección aórtica aguda en departamento de emergencias
}

\section{An atypical presentation of acute aortic dissection in the emergency department}

\author{
Freille DG ${ }^{1}$, Álvarez Valdés BA ${ }^{1}$, Nahuelan $\mathrm{AC}^{1}$, Martínez Mangini AP ${ }^{1}$.
}

\begin{abstract}
Resumen La disección de un aneurisma aórtico es una emergencia cuyo pronóstico depende de un diagnóstico rápido y certero. Se caracteriza por la disección de los planos laminares de la media, con la formación de un canal dentro de la pared aórtica, que con frecuencia puede causar una hemorragia masiva.

Los síntomas clásicos son: dolor intenso y repentino de pecho que se extiende hacia el cuello o la espalda, disnea y síntomas neurológicos tales como pérdida de visión, debilidad o parálisis de un lado del cuerpo.

La mortalidad de la disección aórtica es alta; según la mayoría de los autores más de un tercio de los pacientes mueren en las primeras 24 horas y casi el $95 \%$ mueren en el primer mes.

Presentamos el caso de un paciente masculino de 81 años de edad con una presentación atípica de disección de aneurisma de aorta torácica atendido por un episodio sincopal recuperado, sin dolor, disnea ni síntomas neurológicos.
\end{abstract}

Palabras claves: síncope, disección de aorta, aneurisma

\begin{abstract}
The dissection of an aortic aneurysm is an emergency whose prognosis depends on a prompt and accurate diagnosis. It is characterized by the dissection of the laminar planes of the media, with the formation of a channel within the aortic wall, which can cause massive hemorrhage.

The classic symptoms are: sudden and severe chest pain that extends to the neck or back, dyspnea and neurological symptoms such as vision loss, weakness or paralysis of one side of the body.

Mortality is high with more than a third of patients dying within the first 24 hours and almost 95\% during the first month.

We present the case of an 81-year-old male patient with an aortic thoracic aneurysm dissection who first presented with a full recovered syncopal episode without pain, dyspnea or neurological symptoms.
\end{abstract}

Keywords: syncope, aortic dissection, aneurysm

1. Servicio de Guardia Central. Clínica Universitaria Reina Fabiola, Universidad Católica de Córdoba Correspondencia: Diego G. Freille. Oncativo 1240 B $^{\circ}$ General Paz - X5004FXS Córdoba, Argentina. Te. 0351-4142121 e-mail: gfreille@hotmail.com 


\section{Introducción}

La disección aórtica es un desafío diagnostico dentro del área de emergencias, se produce generalmente de forma brusca, pero no es infrecuente la forma crónica. El síntoma más frecuente es el dolor torácico (78-90\%) 1,2.

Dos tercios de las disecciones de aorta afectan a la aorta ascendente (Stanford tipo A), y generalmente el sitio de disección inicial se encuentra en este lugar. El otro tercio afecta sólo a aorta descendente (Stanford tipo B), siendo el sitio inicial de disección más frecuente la raíz de arteria subclavia izquierda. Otras localizaciones del inicio de disección (arco aórtico, abdominal) son más raras, aunque posibles 3 .

Existen dos teorías sobre el evento inicial de la disección de la íntima: un desgarro primario de la íntima o una hemorragia de la vasavasorum que debilita la pared y rompe la íntima 4 . La hipertensión arterial es el factor más frecuentemente asociado a la disección aórtica, en la mayoría de las series está presente del 70 al $90 \%$ de los pacientes, predominantemente en disección tipo B. La hipertensión arterial acelera el proceso degenerativo normal de la capa media. Otros factores asociados son las anormalidades del tejido conectivo, la presencia de aorta bicúspide y coartación aórtica 5 .

Los pacientes con disección aórtica sin dolor que se presentan con una alteración de la conciencia o un déficit neurológico tienen una mayor morbilidad y peor pronóstico que los pacientes con presentaciones típicas, probablemente debido a que estén sub diagnosticados.6

Una vez realizada la anamnesis y exploración física detallada, y ante la sospecha de disección aórtica, es necesario realizar las pruebas complementarias capaces de descartar o confirmar el diagnóstico de la misma. Además de la radiología simple de tórax, también se utilizan la tomografía axial computarizada y ecocardiografía Doppler 7,8.

\section{Caso Clínico:}

Paciente de sexo masculino de 81 años de edad, con antecedentes de dislipemia e hipertensión arterial. El paciente se encontraba medicado con enalapril $10 \mathrm{mg}$ por día e hidroclorotiazida $25 \mathrm{mg}$ por día.
El paciente consultó en guardia central por haber presentado en su domicilio un episodio sincopal con vómitos alimenticios y relajación de esfínteres, con posterior recuperación adintegrum, negando dolor y refiriendo astenia.

Al examen físico se presentó con presión arterial $80 / 50 \mathrm{mmHg}$, frecuencia cardiaca de $90 \mathrm{lpm}$, saturación de oxígeno $=97$. Los ruidos cardíacos se auscultaban hipofonéticos y no se auscultaron soplos ni latidos patológicos. Se realizó un examen neurológico que resultó normal y se observó un traumatismo craneoencefálico con herida cortante que requirió sutura.

El electrocardiograma mostró ritmo sinusal, sin cambios isquémicos agudos ni rectificación del segmento ST. Las pruebas de laboratorio arrojaron los siguientes resultados: glóbulos blancos $22.000 / \mathrm{mm} 3$ con $87 \%$ de neutrófilos, hemoglobina 15g/dL, CPK 262 U/l (menor a 170 U/I), CPK MB 25 U/l (menor al 4\% de CPK). El resto de la analítica fue normal. Una radiografía de tórax evidenció un ensanchamiento del mediastino (Figura 1). Por este hallazgo se realizó una angiotomografía de tórax que reveló un aneurisma con disección de aorta ascendente hasta la raíz del tronco braquio-cefálico (Figura 2).

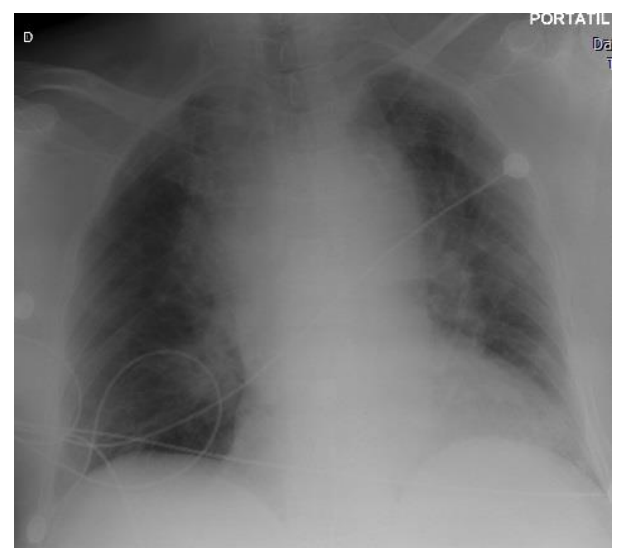

Figura 1 - Radiografía de tórax: Mediastino ensanchado con botón aórtico prominente y desviación de aorta descendente.

Se decide hospitalizar al paciente en unidad coronaria para preparación de cirugía reparadora con colocación de prótesis. 


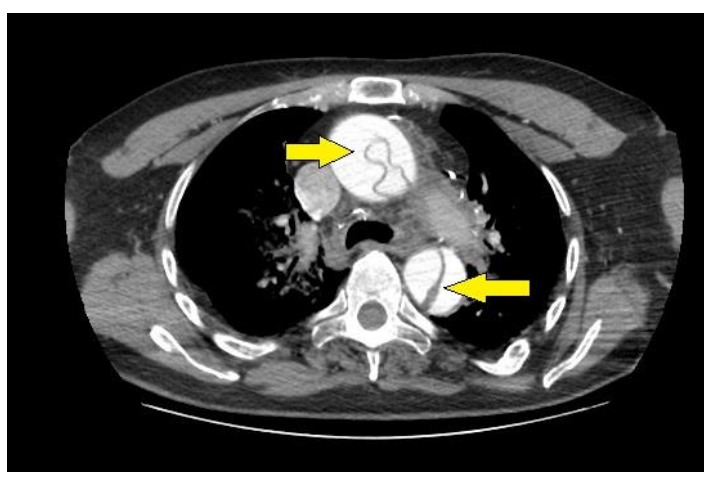

Figura 2. Angiotomografía de tórax - Disección de aorta ascendente y descendente tipo A de Stanford con un flap de la íntima que se extiende a lo largo de la pared formando un falso lumen

\section{Discusión}

Si bien el síntoma inicial más frecuente de presentación de la disección aórtica es el dolor torácico $(58-90 \%)$, este caso denota la necesidad de reconocer las manifestaciones menos frecuentes de las disecciones de aorta torácica para llegar a un diagnóstico precoz. El síncope es el síntoma de presentación en menos del $5 \%$ de los casos y sólo en $1 \%$ de los casos lo es el síncope sin otros síntomas concomitantes 9 .

El síncope en Argentina es un motivo frecuente de consulta en guardias y servicios de emergencia, representando el $1-3 \%$ de las consultas. Un $18.5 \%$ de la población general sufre un síncope alguna vez en su vida10. Las etiologías de este cuadro son muy variadas, algunas veces benignas, y otras veces graves, por lo que a menudo suponen un desafío diagnóstico11. Por ello se han hecho esfuerzos para desarrollar evaluadores pronósticos que ayuden al tratamiento de este cuadro. Es importante detectar con la mayor exactitud posible a aquellos enfermos cuyo pronóstico sea grave. Estos evaluadores pronósticos contemplan factores como el historial de insuficiencia cardiaca congestiva, hematocrito, electrocardiograma anormal, historia de disnea y presión arterial sistólica12. Uno de los scores más extendidos es el San Francisco Syncope Rules, en que la presencia de cualquiera de los contemplados supone la inclusión del enfermo en el grupo de riesgo elevado, con un pronóstico de padecer evento grave en los próximos siete días, con una sensibilidad $98 \%$ y especificidad del $56 \% 13$.

Es importante realizar una correcta anamnesis y solicitar los estudios a los pacientes que consultan en guardia o departamentos de emergencia, incluyendo electrocardiograma $\mathrm{y}$ laboratorio que son estudios de bajo costo y alta rentabilidad. En caso de presentar antecedentes o predictores de riesgo para disección aórtica se debe solicitar con urgencia eco cardiograma o angiotomografía de tórax.

La prevalencia de disección de aorta asociada a síncope sin otras manifestaciones es baja, pero con una alta mortalidad lo que hace que un diagnóstico acertado cambie completamente el pronóstico del paciente.

\section{Conclusión}

Debe considerarse la posibilidad de disección aórtica en el diagnóstico diferencial de síncope, aún cuando no exista un compromiso multiorgánico. El diagnóstico debe realizarse lo más rápido posible, dependiendo del paciente y de los medios disponibles en el centro receptor ya que demoras innecesarias aumentarían la morbimortalidad del paciente.

\section{Bibliografía}

1. Hagan $P$, Nienaber $C$, Isselbacher C, Bruckman D, Karavite D, Russman P, et al. The International Registry of Acute Aortic Dissection. JAMA. 2000; 283: 897-903.
2. Rico A, Lucena J, Blanco M, Marín R, Barrero E, Santos M. Muerte súbita por disección de aorta torácica secundaria a coartación de aorta. Cuadernos de Medicina Forense. 2008;(51), 55 60 
Freille DG, Álvarez Valdés BA, Nahuelan AC, Martínez Mangini AP. Una presentación atípica de disección aórtica aguda en departamento de emergencias

3. Olivert Cruz M, Romero Cabrera

AJ, Bembibre Taboada R,

Bermúdez López J. Disección

aórtica: Estudio en un decenio

(1987-1997). Revista Cubana de

Medicina. . 2000; 39(4), 217-

221

4. Contreras Zúñiga E, Zuluaga Martínez SX, Gómez Mesa J E, Ocampo Duque V, Urrea Zapata CA. Disección aórtica: estado actual. Revista Costarricense de Cardiología, 2009; 11(1), 19-27.

5. Ros-Díe E, Fernández-Quesada F, Ros-Vidal R, Salmerón-Febres LM, Linares-Palomino JP, SellésGaliana, F. Historia natural de la disección aórtica. Angiología. 2006; 58:S59-S67.

6. Imamura $\mathrm{H}$, Sekiguchi $\mathrm{Y}$, Iwashita T, Dohgomori $\mathrm{H}$, Mochizuki K, Aizawa K,

Okamoto K. Painless acute aortic dissection. Circulation Journal, 2011; 75: 59-66.

7. Zamorano JL, Mayordomo J, Evangelista A, San Román JA, Bañuelos C, Gil M. Guías de práctica clínica de la Sociedad Española de Cardiología en enfermedades de la aorta. RevEspCardiol. 2000; 53:531541.

8. Selman R, Kursbaum A, Ubilla $\mathrm{M}$, Turner E, Espinoza C
Espinoza J, Oppliger E. Disección aórtica tipo A: Resultados operatorios y seguimiento a mediano plazo. Revista médica de Chile, 2010 138: 982-987.

9. Guideline for the evaluation and management of patients with syncope. Journal of the American College of Cardiology. 2017; 70(5) 39.

10. MuiñoMiguez A, García V López González C, Gómez Antúnez M, Ortiz Vega M, Ortiz Alonso J. Disección aórtica aguda. Anales de Medicina Interna. Arán Ediciones. 2002 abr. 19(4):19-23

11. Sociedad Argentina de Cardiología. Consenso para el diagnóstico y tratamiento del síncope. Revista Argentina de Cardiología. 2016; 80(1) 69-90.

12. Quinn JV, Stiell IG, McDermott DA, et al. Derivation of the San Francisco Syncope Rule to predict patients with short-term serious outcomes. Ann Emerg Med. 2004; 43:224-232.

13. Schladenhaufen R, Feilinger $S$, Pollack M, et al. Application of San Francisco Syncope Rule in elderly ED patients. Am J Emerg Med. 2008; 26:773-778. 